\title{
Correlation-induced single-flux-quantum penetration in quantum rings
}

\author{
A. J. M. Giesbers ${ }^{1}$, U. Zeitler ${ }^{1 \star}$, M. I. Katsnelson ${ }^{2}$, D. Reuter ${ }^{3}$, A. D. Wieck ${ }^{3}$, G. Biasiol ${ }^{4}$, L. Sorba ${ }^{4,5}$ \\ and J. C. Maan'
}

The confined electronic states of mesoscopic structures in a magnetic field are arranged in Landau levels consisting of spatially discrete eigenstates. These Landau orbits are the quantum mechanical analogue of classical cyclotron orbits. Here we present magnetoconductance oscillations in semiconductor rings, which visualize the spatial discreteness of the Landau orbits in high magnetic fields (typically B $>2 \mathrm{~T}$ ). We will show that these oscillations are caused by the fluxquantized, discrete electronic size of the ring leading to a corresponding modulation of its two-point conductance. The oscillation period is given by the number of flux quanta penetrating the conducting area of the structure. These high-field oscillations are distinctively different from the well-known Aharonov-Bohm effect ${ }^{1}$, where, most generally, the penetration of individual flux quanta $h / e$ through a nanostructure causes periodic crossings of field-dependent energy levels, which give rise to magneto-quantum oscillations in its conductance ${ }^{2-4}$.

Conductance oscillations in mesoscopic structures subjected to a magnetic field are generally assigned to periodic modifications of the electron phase with the magnetic flux penetrating the system. When a charged particle moves phase-coherently through a quantum ring $(\mathrm{QR})$, it accumulates a phase, which changes periodically with the number of magnetic flux quanta, $\phi_{0}=h / e$, enclosed by the ring (Fig. 1b). This results in magnetic-fieldperiodic oscillations of its conductance ${ }^{1-4}$.

Such traditional Aharonov-Bohm (AB) oscillations are also observed in high magnetic fields in QRs (refs 5,6) and quantum $\operatorname{dots}^{7-9}$. They are due to quantum Hall edge channels propagating along the outer rim and their period is given by the area encompassed by this edge. This means that changing a quantum dot to a QR by removing its centre does not affect the period of the $\mathrm{AB}$ oscillations. However, in narrow rings, where inner and outer edge channels are no longer separated, phase coherence is destroyed and the $\mathrm{AB}$ oscillations disappear in strong magnetic fields.

Generally, any field dependence of the single-particle energies in a magnetic field can lead to a redistribution of electrons and will cause field-dependent oscillations in the physical properties of the system. Specifically in quantum dots ${ }^{10,11}$, oscillations arising from crossings of different Landau levels are observed. They are periodic only when just the two lowest Landau levels are occupied, and these oscillations disappear in the quantum limit when only one Landau level is filled.
We have fabricated four QR samples (see Fig. 1a and Methods for details of the sample fabrication) with different ratios between the insulating island in the centre of the ring and its conducting rim.

In low magnetic fields these $\mathrm{QRs}$ show pronounced $\mathrm{AB}$ oscillations in their magnetoconductance (Fig. 1c) with a period $\Delta B=\phi_{0} / A . A=(1 / 4) \pi l d_{\mathrm{AB}}^{2}$ is the area enclosed by the current paths and $d_{\mathrm{AB}}$ are the average ring diameters deduced from the measured oscillation periods, which indeed agree reasonably with the lithographically defined dimensions (Table 1). The presence of $\mathrm{AB}$ oscillations in our QRs proves that, at low fields, electron waves move coherently through them. Part of the phase information is lost, however, by inelastic-scattering events inside the ring and in the quantum point contacts, leading to an oscillation amplitude that is smaller than the conductance quantum $e^{2} / h$. Indeed, for the largest ring (sample 4), nearly all phase coherence is destroyed and only weak $\mathrm{AB}$ oscillations remain visible.

At higher magnetic fields, $1 / B$-dependent Shubnikov-de-Haas oscillations superimposed on the $\mathrm{AB}$ oscillations appear, and finally the $\mathrm{AB}$ oscillations disappear completely ${ }^{3,6,12}$. However, when carefully tuning the in-plane gate voltages another type of fieldperiodic oscillation appears in high magnetic fields. Subsequently we shall refer to these as high-field oscillations. Figure $2 \mathrm{~b}$ shows an intermediate regime for sample 1 . The voltage on the two gates (see Fig. 2a) is tuned such that both the $\mathrm{AB}$ oscillations and the high-field oscillations are visible in one field sweep. For this sample, $\mathrm{AB}$ oscillations are visible up to about $0.3 \mathrm{~T}$, and high-field oscillations appear above approximately $1.4 \mathrm{~T}$, with a period that is considerably larger than that of the low-field $\mathrm{AB}$ oscillations. These oscillations persist with a constant period in the entire quantum Hall regime, in particular also when only the lowest Landau level is occupied $(v<1)$. This makes them crucially different from oscillations observed in quantum dots ${ }^{10,11}$, which are caused by the redistribution of electrons between different levels inside a nanostructure. Such oscillations are periodic only in the regime $1<v<2$; for higher filling factors they become more and more irregular. Moreover, oscillations caused by magnetic depopulation of individual Landau levels disappear when only one Landau level is filled, which is not the case for the high-field oscillations we observe.

The different nature of our high-field oscillations compared with traditional AB type oscillations is explained further in Fig. 2c, where we show the evolution of the two oscillation periods for sample 1 as a function of gate voltage. At high voltages, the ring is open and $\mathrm{AB}$ oscillations with a period of $80 \mathrm{mT}$ (red circles) appear at low

\footnotetext{
${ }^{1}$ High Field Magnet Laboratory, Institute for Molecules and Materials, Radboud University Nijmegen, Toernooiveld 7, 6525 ED Nijmegen, The Netherlands, ${ }^{2}$ Theory of Condensed Matter, Institute for Molecules and Materials, Radboud University Nijmegen, Heyendaalseweg 135, 6525 AJ Nijmegen, The Netherlands, ${ }^{3}$ Lehrstuhl für Angewandte Festkörperphysik, Ruhr-Universität Bochum, Universitätstraße 150, 44780 Bochum, Germany, ${ }^{4}$ Laboratorio Nazionale TASC INFM-CNR, Area Science park, 34012 Trieste, Italy, ${ }^{5}$ NEST INFM-CNR and Scuola Normale Superiore, 56126 Pisa, Italy. *e-mail:U.Zeitler@science.ru.nl.
} 
a

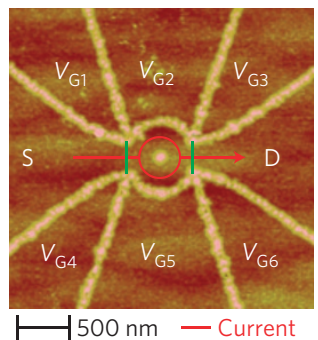

b

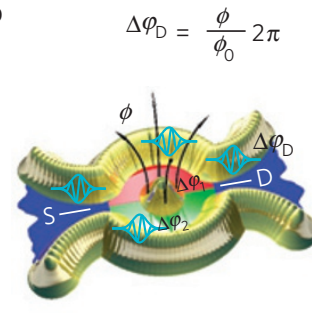

C

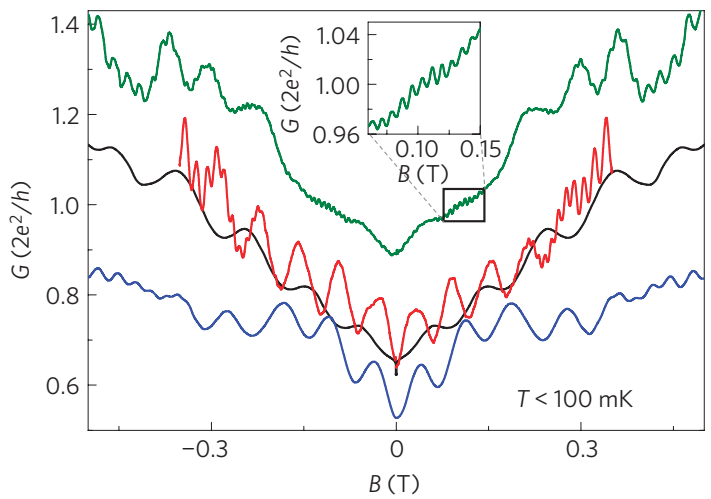

Figure 1 | AB effect in a QR. a, Atomic force micrograph of a $Q R$. The bright lines and the bright dot are oxides that define the ring (red line) in the 2DES underneath. The in-plane gates $\left(V_{\mathrm{G} 1}, V_{\mathrm{G} 4}\right.$ and $\left.V_{\mathrm{G} 3}, V_{\mathrm{G} 6}\right)$ are used to tune the quantum point-contacts at the entrance and exit (indicated by the vertical green lines). The middle in-plane gates $\left(V_{\mathrm{G} 2}\right.$ and $\left.V_{\mathrm{G} 5}\right)$ tune the electron concentration in both arms of the ring. $\mathbf{b}$, Schematic representation of a QR with flux through the centre and the two different phases accumulated in both arms of the ring, leading to a phase difference $\Delta \varphi_{D}$ at the drain that is periodic with the number of flux quanta penetrating the entire ring. c, Typical measurements of the $A B$ oscillations for four QRs fabricated on different samples (temperature $T<100 \mathrm{mK}$ ). The periods of the oscillations, $80 \mathrm{mT}$ (blue, sample 1), $60 \mathrm{mT}$ (red, sample 2), $100 \mathrm{mT}$ (black, sample 3) and $8 \mathrm{mT}$ (green, sample 4), correspond to electronic diameters $d_{A B}$ which are in reasonable agreement with the lithographically defined ring diameters $d_{\text {litho }}$ (see Table 1). The fast oscillations at higher magnetic fields are 1/B-periodic Shubnikov-de Haas oscillations.

Table 1 | Dimensions of the four samples investigated.

\begin{tabular}{|c|c|c|c|c|c|c|}
\hline No. & $\begin{array}{l}d_{\text {litho }} \\
(\mathrm{nm})\end{array}$ & $\begin{array}{l}w_{\text {litho }} \\
(\mathrm{nm})\end{array}$ & $\begin{array}{l}\Delta B_{A B} \\
(m T)\end{array}$ & $\begin{array}{l}d_{\mathrm{AB}} \\
(\mathrm{nm})\end{array}$ & $\begin{array}{l}\Delta B_{\mathrm{HF}} \\
(\mathrm{mT})\end{array}$ & $\begin{array}{l}w \\
(\mathrm{~nm})\end{array}$ \\
\hline 1 & 250 & 200 & 80 & 260 & $\begin{array}{l}104 \\
158\end{array}$ & $\begin{array}{l}51 \\
33\end{array}$ \\
\hline 2 & 300 & 200 & 60 & 300 & $\begin{array}{l}163 \\
185\end{array}$ & $\begin{array}{l}27 \\
24\end{array}$ \\
\hline 3 & 250 & 200 & 100 & 230 & 126 & 42 \\
\hline 4 & 800 & 240 & 8 & 810 & $\begin{array}{l}53 \\
60\end{array}$ & $\begin{array}{l}31 \\
27\end{array}$ \\
\hline
\end{tabular}

Comparison of the lithographically defined average diameter, $d_{\text {litho, }}$, with an average ring diameter $d_{A B}$ as deduced from the AB-oscillation period $\Delta B_{A B}=4 \phi_{0} / \pi d^{2}$. The period of the high-field oscillations, $\Delta B_{\mathrm{HF}}=\phi_{0} / \pi d w$ is modelled with a flux-penetrating area of a conducting ring with a diameter $d=d_{A B}$ and width $w$. The values for $\Delta B_{H F}$ of samples 1, 2 and 4 span the possible ranges of gate voltages where we were able to observe high-field oscillations (see Fig. 2c)

magnetic fields (right inset). Lowering the gate voltage squeezes the ring, effectively pinching off the ring (hatched red area, centre inset). This squeezing has no significant effect on the $\mathrm{AB}$ period;

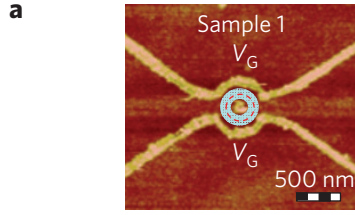

b

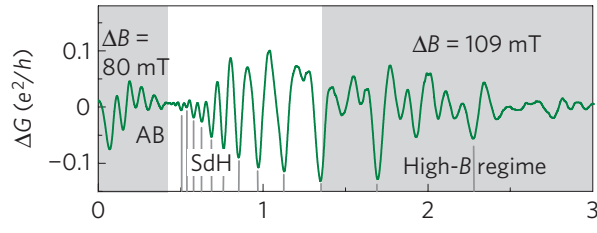

$B(T)$

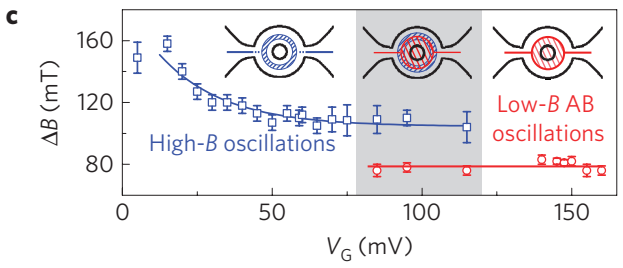

Figure 2 | Conductance oscillations in sample 1 as a function of gate voltages. a, AFM micrograph of sample 1 with two gates serving as a plunger gate and controlling the point-contacts simultaneously.

b, Quantum oscillations in an intermediate regime where the gate voltage ( $V_{\mathrm{G}}=95 \mathrm{mV}$ on both gates) is tuned such that both the Aharonov-Bohm oscillations $(B<0.4 \mathrm{~T})$ and the high-field oscillations $(B>1.4 \mathrm{~T})$ are visible in one field sweep. The $1 / B$-periodic oscillations starting around $B=0.4 \mathrm{~T}$ (indicated by the vertical grey lines) are due to the Shubnikov-de-Haas oscillations in the leads. c, Period of the low-field AB oscillations (red circles) and the high-field oscillations (blue squares) as a function of gate voltage applied to both in-plane gates (the lines are a guide to the eye). The error bars represent the standard deviation of the average period measured in the low-field interval and the high-field interval of Fig. 2b. At high gate voltages the ring is open and $A B$ oscillations with a period of $\Delta B=80 \mathrm{mT}$ are visible. The right inset sketches an open conducting channel through the ring at high gate voltages (red line) and the flux-penetrated area (red hatched) responsible for $A B$ oscillations with a period of $\Delta B=80 \mathrm{mT}$. When the gate voltage is lowered, oscillations with a period $\Delta B>100 \mathrm{mT}$ start to appear in high magnetic fields. This period corresponds to a flux-penetrated area given by the conducting parts of the ring (blue hatched, middle inset). This area shrinks further with decreasing gate voltages (left inset), which leads to an increase in the related period. For the lowest gate voltages $\left(V_{g}<0 \mathrm{~V}\right)$, the blue area is completely pinched off and the oscillations disappear.

it only reduces the oscillations' amplitude until they completely disappear. In this transition region, $80 \mathrm{mV}<V_{\mathrm{G}}<120 \mathrm{mV}$, the high-field oscillations, with a larger period, start to appear above $B=1.5 \mathrm{~T}$ (filling factor $v<8$ ). For the lowest gate voltages $\left(V_{\mathrm{G}}<80 \mathrm{mV}\right), \mathrm{AB}$ oscillations are no longer visible and only the high-field oscillations survive. Finally, the ring is completely pinched off and all oscillations disappear.

We also observed $B$-periodic high-field oscillations in the three other samples; results are shown in Fig. 3. They persist deep into the quantum limit $(v<1)$, with a period that is independent of the magnetic field (see also Supplementary Fig. S1), which rules out the possibility that the oscillations are due to magnetic depopulation of Landau levels as observed in quantum dots ${ }^{10,11}$. Their significantly larger period compared with traditional $A B$ oscillations is determined by a flux-penetrated area considerably smaller than the average surface of a circular path through the ring. This effect is most pronounced for the largest ring, where the corresponding period is enlarged by nearly an order of magnitude. Evidently, the observed periods no longer fit with the number of flux 

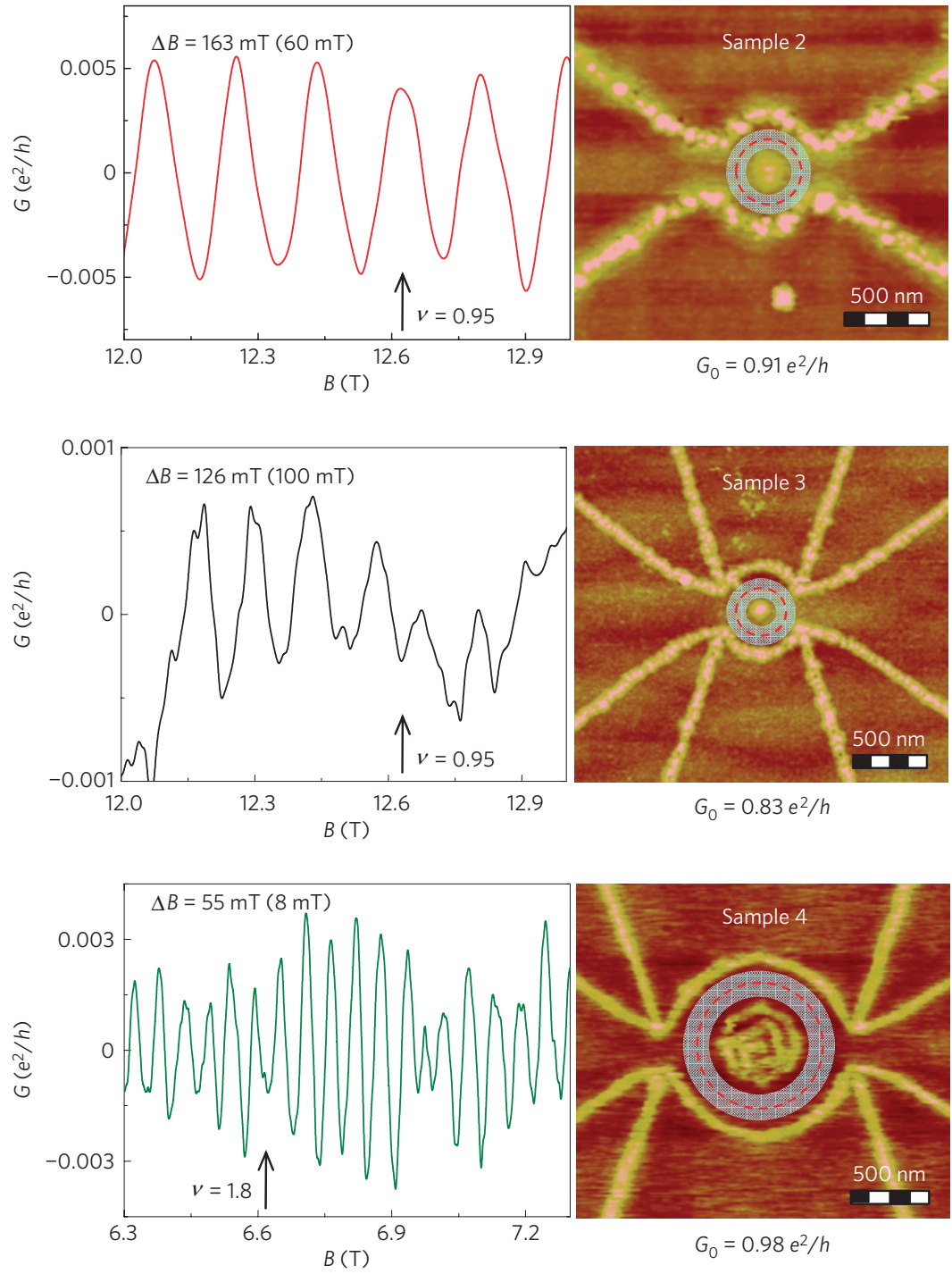

Figure 3 | High-field oscillations for samples 2, 3 and 4. The oscillation periods $\Delta B_{H F}$ are compared with the corresponding $A B$ periods in the parentheses (Fig. 1). The arrows indicate the filling factor $v$ at a particular point. $G_{0}$ is the conductance of the ring at zero magnetic field at the measured gate-voltage setting, showing that the point contacts to the ring were semi-open (that is, far away from the Coulomb-blockade regime). The gate-voltage and field dependence of the conductance for sample 1 are shown in Supplementary Fig. S2. The right panels show AFM micrographs of the structures; the grey areas represent the conducting part of the ring.

quanta penetrating the average area inside a ring. Instead, we find that the period is given by the number of flux quanta penetrating the conducting ring-shaped grey areas as sketched in the right panels of Fig. 3: $\Delta B_{\mathrm{HF}}=\phi_{0} /(\pi d w)$.

The results for all samples are summarized in Table 1, where the ring widths $w$, as estimated from $\Delta B_{\mathrm{HF}}$, are listed. The widths, $25-50 \mathrm{~nm}$, are indeed in reasonable agreement with the lithographic width when edge depletion of around $50-100 \mathrm{~nm}$ is taken into account ${ }^{13,14}$. The ring width can be reduced by applying a more negative in-plane gate voltage, which indeed leads to an increase in the observed high-field oscillation period (see Fig. 2c).

Within a framework of traditional $\mathrm{AB}$ oscillation, our findings that the oscillation period is given by the conducting area rather than the total size of the ring would suggest that the electrons move through a phase-coherent path along counter-propagating edge channels at the inner edge and the outer edge, which are connected at one point. In this respect, they are similar to long-period oscillations observed in quantum dots, where electrons scattered from one edge channel to another pick up an extra $A B$ phase ${ }^{15,16}$. In our case, electrons transferring a ring through such a path defined by an inner and outer edge pick up a phase, which is determined by the grey area in the right panels of Fig. 3. Though we consider such a scenario not very probable, we cannot honestly rule out this possibility from the experimental point of view.

In the following we shall propose an alternative explanation on the basis of the discreteness of the electronic states and the Coulomb interaction between them. For simplicity, we model our ring by a strip with length $L_{y}=\pi d$, using periodic boundary conditions in the (azimuthal) $y$ direction. The confinement in the (radial) $x$ direction is described by a parabolic potential $V(x)=1 / 2 m^{*} \omega_{0}^{2} x^{2}$, where $m^{*}=0.067 m_{\mathrm{e}}$ is the effective mass of the electrons in GaAs and $\omega_{0}$ is the angular frequency defining the harmonic potential. The eigenenergies of this system in a quantizing magnetic field are

$$
E_{n, k_{y}}=\left(n+\frac{1}{2}\right) \hbar \omega+\frac{\hbar^{2} k_{y}^{2}}{2 m^{*}} \frac{\omega_{0}^{2}}{\omega^{2}}
$$

with $n=0,1,2, \ldots$ the Landau-level index and $\omega^{2}=\omega_{0}^{2}+\omega_{c}^{2}$; $\omega_{\mathrm{c}}=e B / m^{*}$ is the cyclotron frequency. The discrete energies of these Landau orbits originate from Landau levels characterized by 


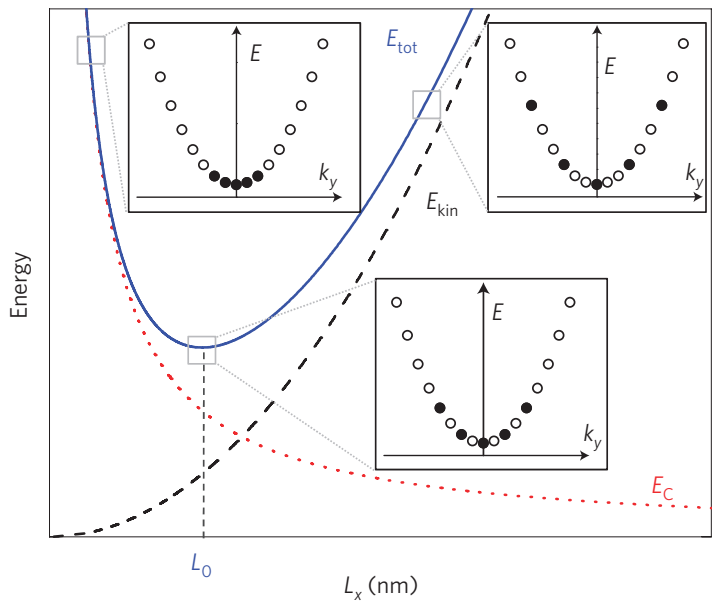

b

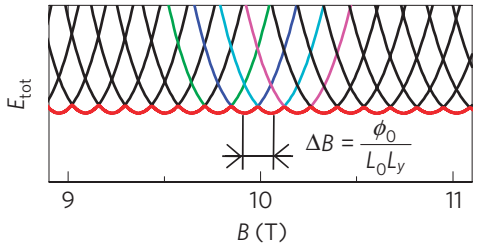

c

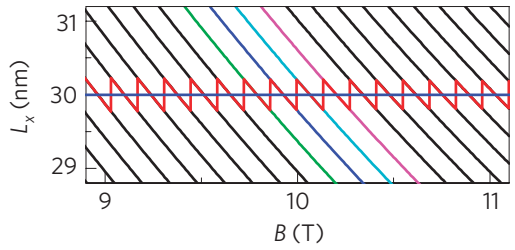

d

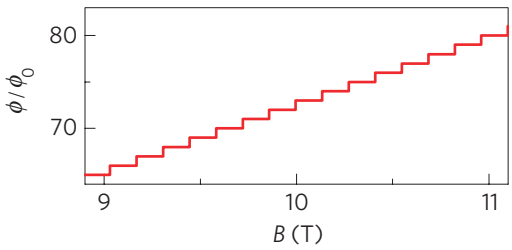

Figure 4 | Model for the oscillating size of an interacting electron system in the quantum limit. a, Sketch of the total energy $E_{\text {tot }}$ (blue line) as a function of the ring size. $E_{\text {tot }}$ is governed by the competition between the single-particle kinetic energy $E_{\text {kin }} \propto L_{x}^{2}$ (black dashed) and the Coulomb energy $E_{C} \propto L_{x}^{-1}$ (red dotted). When all electrons are in the lowest energetic states (left inset), the Coulomb repulsion will dominate the total energy of the system. Enabling the electrons to distribute over higher-energy states reduces the total energy as the Coulomb repulsion is reduced until the minimal-energy configuration is reached at the equilibrium size $L_{0}$ (centre inset). For larger inter-electronic distances, the single-particle energy dominates the total energy (right inset). $\mathbf{b}$, Total energy of a model ring with width $L_{0}=30 \mathrm{~nm}$ and circumference $L_{y}=\pi d=1 \mu \mathrm{m}$, filled with 50 electrons for $M=67-80$. $M$ is the possible number of Landau orbits inside the structure. The red line depicts the minimal energy as a function of magnetic field, leading to oscillations in the energy with a period $\Delta B=\phi_{0} /\left(L_{0} L_{y}\right)$. c, Electronic width of the ring (red line) oscillating around its ideal width $L_{0}$ (blue line) with the same frequency. The actual ring size follows possible widths $L_{M}$ (diagonal lines) as close as possible to $L_{0}$. $\mathbf{d}$, Quantized flux penetration into the conducting area of our model ring. Magnetic flux quanta enter the ring one by one whenever it adapts its size abruptly from $L_{M}$ to $L_{M+1}$.

a Landau-level index $n$ and the quantization of the wavevector $k_{y}=k 2 \pi / L_{y}$ (with $k= \pm 1, \pm 2, \ldots$ ) caused by the periodic boundary conditions in the (azimuthal) $y$ direction. In a magnetic field, the $k_{y}$ coordinate couples to the real-space coordinate $x$ through the Landau gauge, leading to a spatial energy dispersion for each Landau level

$$
E_{n}\left(x_{k}\right)=\left(n+\frac{1}{2}\right) \hbar \omega+\frac{1}{2} m \frac{\omega_{0}^{2} \omega_{\mathrm{c}}^{2}}{\omega^{2}} x_{k}^{2}
$$

with Landau orbits centred at $x_{k}=l_{B}^{2} k_{y}=k 2 \pi l_{B}^{2} / L_{y}\left(l_{B}=\sqrt{h / e B}\right.$ is the magnetic length). Without electron-electron interaction, the electrons fill the lowest-lying states (Fig. 4a, left inset) and the electronic width of our model strip is $L_{x}=(N-1) 2 \pi l_{B}^{2} / L_{y}$, where $N$ is the number of electrons in the ring. However, when including Coulomb interaction, it becomes energetically favourable to increase the electronic width of the ring by occupying energetically higher-lying states. The increase of the single-particle energy is compensated by a reduction of the charging energy (Fig. 4a, centre inset). For larger inter-electronic distances the single-particle energy will dominate the total energy (Fig. 4a, right inset).

The total energy $E_{\text {tot }}$ is the sum of the single-particle energy, $E_{\text {kin }}$, and the charging energy, $E_{C}$. In the limit of high magnetic fields where $\omega_{c} \gg \omega_{0}$ and only one $(n=0)$ Landau level is occupied, $E_{\text {kin }}$ for a strip with an electronic width $L_{x}$ containing $N$ electrons can easily be determined by integrating equation (1) with a constant density $\rho(x)=N /\left(L_{x} L_{y}\right)$ :

$$
E_{\text {kin }}=L_{y} \int_{-L_{x} / 2}^{L_{x} / 2} E_{0}(x) \rho(x) \mathrm{d} x \propto L_{x}^{2}
$$

Here we omit a constant offset of the Landau energy, which does not depend on the system size.
The charging energy, $E_{\mathrm{C}}=(\mathrm{Ne})^{2} / 2 \mathrm{C}$, is determined by the capacitance $C$ of the ring to its environment. Most generally, $C$ is decreasing with decreasing size $L_{x}$. Ignoring the capacitances between ring and electrodes/gates ${ }^{17}$, we can illustratively approximate $C$ by the ring's self-capacitance alone, $C \propto L_{x}$, and its total energy is:

$$
E_{\mathrm{tot}}\left(L_{x}\right)=\alpha L_{x}^{2}+\beta L_{x}^{-1}
$$

$\alpha$ and $\beta$ are numeric constants depending on the details of the confinement and the magnitude of the Coulomb interaction. The system minimizes its total energy by adopting an ideal width $L_{0}=(\beta / 2 \alpha)^{1 / 3}$ (see Fig. 4a). Note that the existence of an ideal width $L_{0}$ does not rely on the simple illustrative form of the total energy in equation (2). In more general terms the single-particle energy will always increase with the size of the system and the Coulomb energy will decrease, leading to an optimal size $L_{0}$ with a minimum total energy.

The discreteness of the Landau orbits in the $x$ direction, however, implies that only discrete electronic widths $L_{M}$ are possible, with

$$
L_{M}=l_{B}^{2} \Delta k_{y}=2 \pi l_{B}^{2} \frac{M}{L_{y}}=\frac{h}{e B} \frac{M}{L_{y}}
$$

$M \geqslant N-1$ is an integer. For each of these given widths, the dependence of the total energy on the magnetic field will then be

$$
E_{\text {tot }}^{(M)}(B)=\frac{\alpha \phi_{0}^{2}}{L_{y}^{2}}\left(\frac{M^{2}}{B^{2}}+2\left(\frac{L_{0} L_{y}}{\phi_{0}}\right)^{3} \frac{B}{M}\right)
$$

$E_{\text {tot }}^{(M)}(B)$ are plotted in Fig. $4 \mathrm{~b}$ for $M=67-80$ for a ring with width $L_{0}=30 \mathrm{~nm}$ and a circumference $L_{y}=\pi d=1 \mu \mathrm{m}$. These 
values are chosen to match the experimentally observed length scales in samples 1-3.

For a given magnetic field, the system minimizes its total energy by choosing an appropriate discrete width $L_{M}$ as close as possible to $L_{0}$, which results in magnetic-field periodic oscillations of both the total energy (red line in Fig. 4b) and the electronic width of the ring (Fig. 4c). The oscillation period can be calculated from the distance between two minima in $E_{\text {tot }}^{(M)}(B)$ and $E_{\text {tot }}^{(M+1)}(B): \Delta B=\phi_{0} /\left(L_{0} L_{y}\right)$. It is solely determined by the number of flux quanta penetrating the conducting area of the ring. In particular, it depends neither on the strength and the form of the confinement, nor on the magnitude of the Coulomb interaction, nor on the electron density. Connecting such a ring with a breathing width to source and drain will inevitably influence its coupling to these electrodes, inducing an oscillation in the conductance of the ring with the same period.

More generally, our model calculations show that we can use the discreteness of Landau orbits in high magnetic fields to measure the penetration of individual flux quanta into a nanostructure, an effect that is fundamentally different for the traditional $\mathrm{AB}$ effect relying on phase-coherent transport. As depicted in Fig. 4d, the nanostructure tries to conserve the number of flux quanta penetrating its conducting area by reducing its size (Fig. 4c). Eventually, a new configuration with exactly one more flux quantum is energetically favourable and an additional integer flux quantum penetrates the ring, resulting an abrupt size-change. Of course the high-field oscillations are still sensitive to thermal smearing of the individual energies, and they indeed start to disappear experimentally at temperatures above $100 \mathrm{mK}$ when the thermal energy exceeds the oscillatory contribution to the total energy in Fig. 4 b.

Our admittedly simple model only considers single-particle states. In a real many-body treatment we would have to minimize the total energy of a linear combination of all possible single-particle occupations. Still, the fact remains that the size of the ring (that is, the position of the outermost Landau orbit) oscillates periodically with the number of flux quanta penetrating the structure.

\section{Methods}

Samples were fabricated using local anodic oxidation with an atomic force microscope $\mathrm{e}^{13,14,18}$ (AFM) on two different high-mobility shallow two-dimensional electron systems (2DESs) in a GaAs/AlGaAs heterojunction ${ }^{19}$. In sample 2 the 2DES is situated $55 \mathrm{~nm}$ below the surface with an electron concentration $n=2.8 \times 10^{15} \mathrm{~m}^{-2}$ and a mobility $\mu=64 \mathrm{~m}^{2} \mathrm{~V}^{-1} \mathrm{~s}^{-1}$. Samples 1,3 and 4 were made from a 40-nm-deep 2DES with $n=3.0 \times 10^{15} \mathrm{~m}^{-2}$ and $\mu=116 \mathrm{~m}^{2} \mathrm{~V}^{-1} \mathrm{~s}^{-1}$.

Locally oxidizing the surface leads to a depletion of the underlying 2DES, which permits the fabrication of complex electronic structures such as quantum point contacts ${ }^{13,14}$, quantum dots ${ }^{20}$ and quantum rings ${ }^{4,21}$.

Figure la shows an AFM micrograph of one of our quantum rings (sample 4). The bright lines are oxide lines with a fully depleted 2DES underneath. Electrons move through the ring (red line) from source (S) to drain (D) through two quantum point contacts at the entrance and the exit (green lines). The coupling to source and drain is regulated by applying a voltage to the in-plane gate electrodes $V_{\mathrm{G} 1}-V_{\mathrm{G} 4}$ and $V_{\mathrm{G} 3}-V_{\mathrm{G} 6}$ (refs $\left.13,14,22\right) . V_{\mathrm{G} 2}$ and $V_{\mathrm{G} 5}$ are used as plunger gates to tune the electron density inside each arm of the ring.

We have fabricated four different rings with different average lithographic diameters $d_{\text {litho }}$ and ring widths $w_{\text {litho }}$; see Table 1 . Owing to edge depletion the actual electronic width of the ring is reduced by typically $100 \mathrm{~nm}$. Two rings (samples 1 and 2) were fabricated with only two gates serving as a plunger gate and controlling the point-contacts simultaneously (Fig. 2a); two other rings (samples 3 and 4) had the full gate configuration.

Two-point transport measurements were made, using standard a.c. techniques in a dilution refrigerator with a base temperature of $40 \mathrm{mK}$ and magnetic fields up to $33 \mathrm{~T}$. More details on the global device characteristics and the operation of the gates in a magnetic field are given in Supplementary Fig. S2.
Received 9 July 2009; accepted 22 December 2009; published online 31 January 2010

\section{References}

1. Aharonov, Y. \& Bohm, D. Significance of electromagnetic potentials in the quantum theory. Phys. Rev. 115, 485-491 (1959).

2. Webb, R. A., Washburn, S., Umbach, C. P. \& Laibowitz, R. B. Observation of h/e Aharonov-Bohm oscillations in normal-metal rings. Phys. Rev. Lett. 54, 2696-2699 (1985).

3. Timp, G. et al. Observation of the Aharonov-Bohm effect for $\omega_{\mathrm{c}} \tau>1$. Phys. Rev. Lett. 58, 2814-2817 (1987).

4. Fuhrer, A. et al. Energy spectra of quantum rings. Nature 413, 822-855 (2001).

5. Ford, C. J. B. et al. Electrostatically defined heterojunction rings and the Aharonov-Bohm effect. Appl. Phys. Lett. 54, 21-23 (1989).

6. Timp, G. et al. Suppression of the Aharonov-Bohm effect in the quantized Hall regime. Phys. Rev. B 39, 6227-6230 (1989).

7. van Wees, B. et al. Observation of zero-dimensional states in a one-dimensional electron interferometer. Phys. Rev. Lett. 62, 2523-2526 (1989).

8. Camino, F. E., Zhou, W. \& Goldman, V. J. Aharonov-Bohm electron interferometer in the integer quantum Hall regime. Phys. Rev. B 72, 155313 (2005).

9. Bird, J. P. et al. Coulomb-blockade of the Aharonov-Bohm effect in GaAs/Al $\mathrm{Ga}_{1-x}$ As quantum dots. Phys. Rev. B 50, 14983-14990 (1994).

10. McEuen, P. L. et al. Transport spectroscopy of a Coulomb island in the quantum Hall regime. Phys. Rev. Lett. 66, 1926-1929 (1991).

11. McEuen, P. I. et al. Self-consistent addition spectrum of a Coulomb island in the quantum Hall regime. Phys. Rev. B 45, 11419-11422 (1992).

12. Ihn, T. et al. Quantum mechanics in quantum rings. Adv. Solid State Phys. 43, 139-154 (2003).

13. Held, R. et al. In-plane gates and nanostructures fabricated by direct oxidation of semiconductor heterostructures with an atomic force microscope. Appl. Phys. Lett. 73, 262-264 (1998).

14. Keyser, U. F. et al. Fabrication of a single-electron transistor by current-controlled local oxidation of a two-dimensional electron system. Appl. Phys. Lett. 76, 457-459 (2000).

15. Ford, C. J. B. et al. Resonant suppression of the quantized Hall effect in ballistic junctions. Phys. Rev. B 43, 7339-7342 (1991).

16. Kirczenow, G. \& Castao, E. Diffraction, phase breaking, and Hall anomalies in quantum dots. Phys. Rev. B 43, 7343-7346 (1991).

17. de Vries, D. K., Stelmaszyk, P. \& Wieck, A. D. Intrinsic and extrinsic capacitances of in-plane-gated transistors. J. Appl. Phys. 79, 8087-8090 (1996).

18. Ishii, M. \& Matsumoto, K. Control of the current in 2DEG channel by oxide wire formed using AFM. Jpn. J. Appl. Phys. 34, 1329-1331 (1995).

19. Giesbers, A. J. M. et al. Aharonov-Bohm effect of quantum Hall edge channels. Physica E 40, 1470-1472 (2008).

20. Lüscher, S. et al. In-plane gate single-electron transistor in GaAlAs fabricated by scanning probe lithography. Appl. Phys. Lett. 75, 2452-2454 (1999).

21. Keyser, U. F. et al. Kondo effect in a few-electron quantum ring. Phys. Rev. Lett. 90, 196601 (2003).

22. Wieck, A. D. \& Ploog, K. In-plane-gate quantum wire transistor fabricated with directly written focused ion-beams. Appl. Phys. Lett. 56, 928-930 (1990).

\section{Acknowledgements}

We would like to thank J. J. Schermer and P. Mulder for their technical support and A. McCollam for her comments on the manuscript. This work is part of the research programme of the Stichting voor Fundamenteel Onderzoek der Materie (FOM), which is financially supported by the Nederlandse Organisatie voor Wetenschappelijk Onderzoek (NWO). Part of this work has also been supported by EuroMagNET under EU Contract RII3-CT-2004-506239. D.R. and A.D.W. thank the DFG SFB491 and SPP1285 as well as the BMBF nanoQUIT for support.

\section{Author contributions}

U.Z. and J.C.M. conceived and designed the experiments; D.R., A.D.W., G.B., L.S. and A.J.M.G. grew the high-quality heterostructures and processed the samples; A.J.M.G. structured the QRs, made measurements and interpreted the data in collaboration with U.Z. and J.C.M.; M.I.K. provided theoretical support; A.J.M.G. and U.Z. co-wrote the manuscript. All authors discussed the results and commented on the manuscript.

\section{Additional information}

The authors declare no competing financial interests. Supplementary information accompanies this paper on www.nature.com/naturephysics. Reprints and permissions information is available online at http://npg.nature.com/reprintsandpermissions. Correspondence and requests for materials should be addressed to U.Z. 